\title{
$1 \quad$ Identifying the high entropy characteristic in La-based metallic
}

Langting Zhang ${ }^{\mathrm{a}}$, Yajuan Duan ${ }^{\mathrm{a}, \mathrm{b}}$, Daniel Crespo ${ }^{\mathrm{b}}$, Eloi Pineda ${ }^{\mathrm{b}}$, Takeshi Wada ${ }^{\mathrm{c}}$, Hidemi Kato ${ }^{c}$, J.M. Pelletierd, Jichao Qiao ${ }^{\mathrm{a},{ }^{*}}$

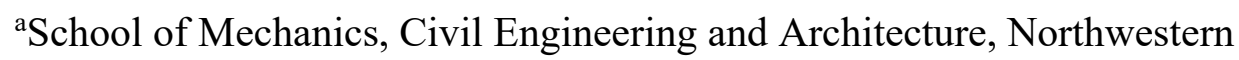

Polytechnical University, Xi'an 710072, China

9 bepartment of Physics, Barcelona Research Center in Multiscale Science and

10 Technology, Institute of Energy Technologies, Universitat Politècnica de Catalunya,

1108019 Barcelona, Spain

12 'Institute for Materials Research, Tohoku University, Sendai 980-8577, Japan

13 dUniversité de Lyon, MATEIS, UMR CNRS5510, Bat. B. Pascal, INSA-Lyon, F-

1469621 Villeurbanne Cedex, France

15 * Corresponding author: Prof. Dr. Jichao Qiao

16 Email: qjczy@nwpu.edu.cn

Abstract: High temperature deformation was probed in a La-based high entropy metallic glass with an evident slow $\beta$ relaxation. The correlation between high configurational entropy and high temperature deformation mechanism was analyzed. On the one hand, by increasing the strain rate, the degree of deviation from Newtonian behavior in high entropy metallic glasses is lower than that of conventional metallic glasses, which is ascribed to the high configurational entropy. On the other hand, high configurational entropy leads to a decrease of activation volume in high temperature deformation of metallic glasses. Identifying the influence of high configurational entropy on the deformation mechanism paves the way for further understanding of the mechanical behavior of metallic glasses.

Keywords: High entropy metallic glass; Slow $\beta$ relaxation; High temperature deformation; Free volume model 
Metallic glasses (MGs) are metallic alloys in which long-range ordered atomic 31 structure is absent ${ }^{1-3}$. High entropy alloys (HEAs) are defined as multicomponent 32 alloys containing at least five elements with equiatomic or near-equiatomic ratio 33 between 5 and 35 at.\% ${ }^{4,5}$. Combining the amorphous structure of MGs and the high 34 configurational entropy of HEAs, high entropy metallic glasses (HE-MGs) have attracted intensive attention in recent years benefiting from their superior mechanical properties, excellent glass-forming ability and unique properties ${ }^{6-8}$. It is significant to investigate the performance of HE-MGs, which could be new candidates to be applied as structural and engineering materials.

The plastic deformation mechanism of MGs can be classified into two types: inhomogeneous deformation and homogeneous deformation ${ }^{9}$. In general, deformation mode of MGs is inhomogeneous at high strain rates and/or low temperature, this mode is characterized by the initiation and propagation of shear bands ${ }^{10}$. On the contrary, homogeneous deformation of MGs takes place at high temperatures (i.e., $T>0.8 T_{g}$, where $T_{g}$ is the glass transition temperature) and/or low strain rates ${ }^{11}$. In the homogeneous mode, MGs display remarkable plasticity and show a smooth transition from Newtonian- (i.e., viscosity independent on strain rate) to non-Newtonian flow (i.e., viscosity dependent on strain rate). This phenomenon has been verified in typical MGs (e.g., Pd-, Cu-, Zr-based MGs) ${ }^{12,13}$. In addition, previous reports have clarified that this transition is attributed to an introduction of structural defects induced by external stress 12. Currently, there is an active discussion relative to the correlation between 51 microscopic deformation mechanisms and relaxation behaviors in glassy solids ${ }^{14-16}$. 52 There are two relaxation processes in glassy solids: the slow $\beta$ relaxation, correlated to local atomic jumps, and the main $\alpha$ relaxation, which is related to large-scale atomic 54 rearrangements ${ }^{17,18}$. Significantly, the slow $\beta$ relaxation is closely correlated to the 55 plasticity of MGs, i.e., the activation energy of slow $\beta$ relaxation $E_{\beta}$ is approximately equal to the energy barrier of shear transformation zones (STZs) $W_{S T Z}{ }^{19}$. 
sluggish diffusion, (3) severe lattice distortion and (4) cocktail effects ${ }^{20}$. However, whether these effects still exist in HE-MGs is still under discussion. Recent investigations have reported great advances in the influence of high configurational entropy on the thermodynamics and kinetics of HE-MGs ${ }^{21-24}$. Benefiting from the sluggish diffusion, the atomic rearrangements of HE-MGs are remarkably hindered ${ }^{25}$. Moreover, the high configurational entropy and the sluggish diffusion improve the structural instability, which results in intensifying the intrinsic ductility ${ }^{24}$. Unfortunately, the deformation mechanism, dynamic relaxation behavior, structural and dynamic heterogeneities and their correlations with high configurational entropy have yet to be clarified. In particular, to what extent the characteristics of HEAs stimulate the plastic deformation mechanism is still unclear. In the current work, $\mathrm{La}_{30} \mathrm{Ce}_{30} \mathrm{Ni}_{10} \mathrm{Al}_{20} \mathrm{Co} 10 \mathrm{HE}-\mathrm{MG}$ was chosen as model alloy to investigate the high temperature plastic deformation mechanism from a novel perspective, considering the high configurational entropy of HE-MGs. The results obtained could offer an ingenious understanding of the apparent features of the high configurational entropy effect.

A master alloy ingot with a nominal chemical composition of $\mathrm{La}_{30} \mathrm{Ce}_{30} \mathrm{Ni}_{10} \mathrm{Al}_{20} \mathrm{Co} 10$ (at.\%) was prepared by arc-melting in a titanium-gettered high purity atmosphere. The ingot was re-melted at least five times to ensure the chemical homogeneity. Ribbons with a cross section of $0.02 \mathrm{~mm} \times 1.2 \mathrm{~mm}$ were prepared by the melt spinning technique. The dynamic mechanical properties of the model glass were characterized in a commercial dynamic mechanical analyzer (DMA, TA instruments Q800) in tension film mode. The strain response $\varepsilon=\varepsilon_{0} \cos (2 \pi f \mathrm{t}+\delta)$ of the viscous-elastic material to a sinusoidal stress $\sigma=\sigma_{0} \cos (2 \pi f \mathrm{t})$ was obtained, where $f$ is the driving frequency and $\delta$ the phase lag. The complex Young's modulus can be expressed as $E=\sigma / \varepsilon=E^{\prime}+i E^{\prime \prime}$, where $E^{\prime}$ is the storage modulus while $E^{\prime \prime}$ is the loss modulus. The internal friction (also called loss factor) correlated to the molecules/atoms mobility of glassy solids is defined as $\tan \delta=E^{\prime \prime} / E^{\prime}$. High temperature deformation tests of model alloys were conducted in the DMA at various temperatures with strain rate ranges from $1 \times 10^{-4} \mathrm{~s}^{-1}$ to $1.25 \times 10^{-3}$ 

$\mathrm{s}^{-1}$.

Fig. 1 (a) displays the temperature dependence of the normalized storage modulus $E^{\prime} / E_{u}$ and loss modulus $E^{\prime \prime} / E_{u}$ of $\mathrm{La}_{30} \mathrm{Ce}_{30} \mathrm{Ni}_{10} \mathrm{Al}_{20} \mathrm{Co} 10 \mathrm{HE}-\mathrm{MG}$ (driving frequency is $3 \mathrm{~Hz}$ and heating rate is $3 \mathrm{~K} / \mathrm{min}$ ). $E_{u}$ is the value of the storage modulus at ambient temperature. One can see that $\mathrm{La}_{30} \mathrm{Ce}_{30} \mathrm{Ni}_{10} \mathrm{Al}_{20} \mathrm{Co}{ }_{10} \mathrm{HE}-\mathrm{MG}$ exhibits a pronounced slow $\beta$ relaxation peak and a main $\alpha$ relaxation peak. The results also show that the temperature span of slow $\beta$ relaxation is wider than that of $\alpha$ relaxation. Fig. 1 (b) shows the normalized loss modulus $E^{\prime \prime} / E^{\prime \prime} \max$ versus the normalized temperature $T / T_{\alpha}$ in typical La-based MGs, where $E^{\prime \prime} \max$ is the maximum value of loss modulus while $T \alpha$ is the $\alpha$ relaxation peak temperature. The interplay between chemical composition and diffusion of the smallest atoms of MGs have a crucial influence on the slow $\beta$ relaxation process ${ }^{26,27}$. As a consequence, the intensity of slow $\beta$ relaxation varies in La-based MGs.
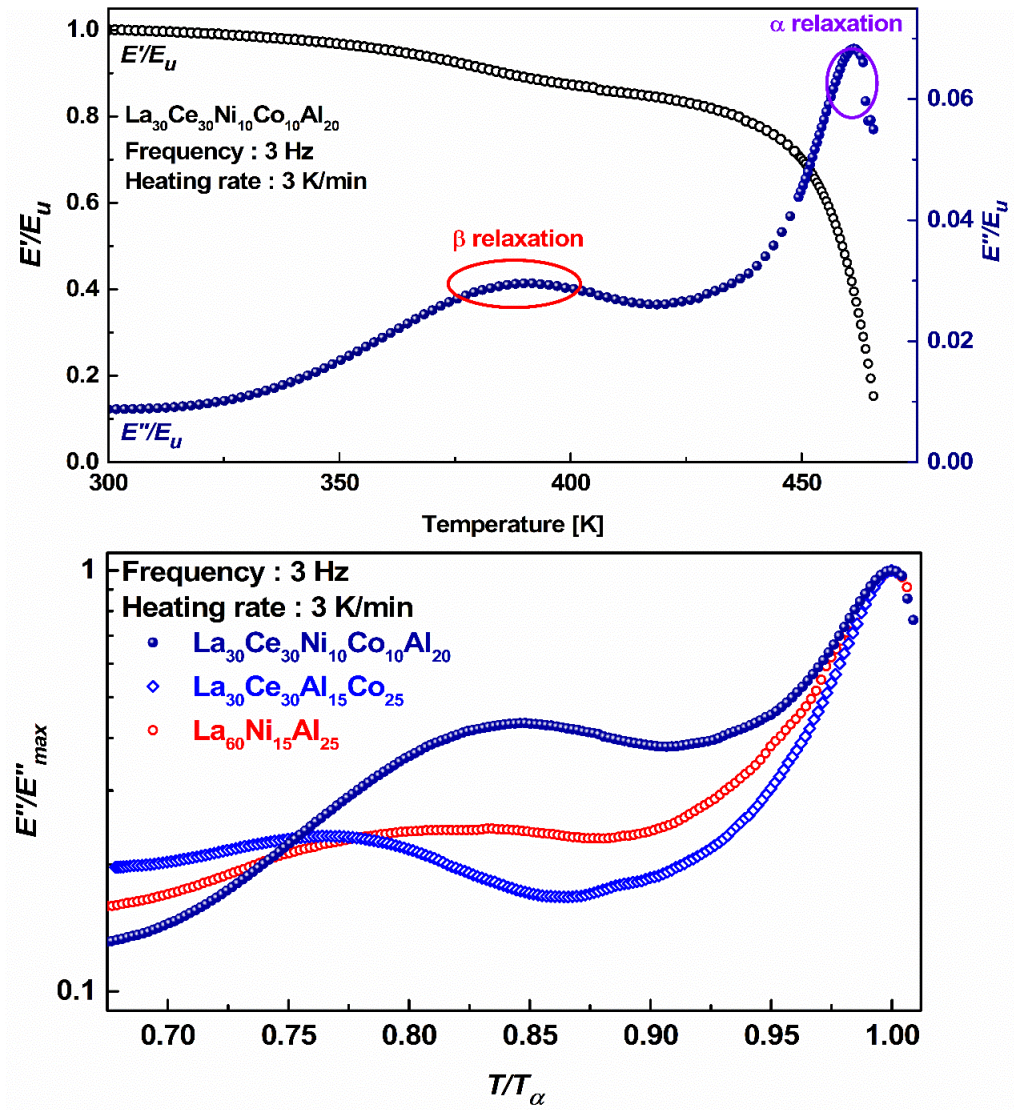

100 Fig.1 (a) Dependence of normalized storage modulus $E^{\prime} / E_{u}$ and loss modulus $E^{\prime \prime} / E_{u}$ of $101 \mathrm{La}_{30} \mathrm{Ce}_{30} \mathrm{Ni}_{10} \mathrm{Al}_{20} \mathrm{Co} 10 \mathrm{HE}-\mathrm{MG}$ on temperature. $E_{u}$ is the value of storage modulus at 102 ambient temperature. (b) Normalized loss modulus $E^{\prime \prime} / E^{\prime \prime}{ }_{\max }$ in typical La-based MGs 103 as function of normalized temperature $T / T_{\alpha} . E^{\prime \prime}{ }_{\max }$ is the maximum of the loss modulus 
and $T_{\alpha}$ is the $\alpha$ relaxation peak temperature.

Strain rate jump tests is an effective method to probe the high temperature deformation behavior of MGs ${ }^{28}$. Fig. 2(a) displays uniaxial, tensile true stress-strain responses during a strain rate jump test at a given temperature of $440 \mathrm{~K}$ and strain rates ranging from $1 \times 10^{-4} \mathrm{~s}^{-1}$ to $1.25 \times 10^{-3} \mathrm{~s}^{-1}$. The true stress increases sharply with the increase of the strain, then, it gradually decreases after reaching a peak value, and finally equilibrates at a stable value. This mechanical behavior can be well described by the free volume model proposed by Spaepen et al ${ }^{29}$. The stress overshoot is attributed to the creation of free volume by plastic deformation ${ }^{30}$.

Based on a series of strain rate jumps, the variation of apparent viscosity with both strain rate and temperature could be readily computed as: $\eta=\sigma(T) / 3 \dot{\varepsilon}$, where $\dot{\varepsilon}$ is the strain rate and $\sigma(T)$ is the corresponding steady state flow stress. Fig. 2(b) shows the apparent viscosity as a function of strain rate at various temperatures. The viscositystrain rate curves of $\mathrm{La}_{30} \mathrm{Ce}_{30} \mathrm{Ni}_{10} \mathrm{Al}_{20} \mathrm{Co}{ }_{10} \mathrm{HE}-\mathrm{MG}$ show the main characteristics of amorphous systems, in which viscosity increases as strain rate decreases and approaches the Newtonian regime. Taking into account that an obvious transition from non-Newtonian to Newtonian flow is hardly observed in such a relatively narrow strain rate window (the lower the strain rate, the longer the isothermal time required, and the more difficult it is for the sample to remain amorphous), at the highest temperature of $453 \mathrm{~K}$, an approximate Newtonian behavior with small strain rate dependence on viscosity could be observed.

Referring to a previous theoretical approach, the evolution of normalized viscosity $\eta / \eta_{N}$ with strain rate can be described by the Kohlrausch-Williams-Watts (KWW) stretched exponential equation ${ }^{31}$ :

$$
\frac{\eta}{\eta_{N}}=\left\{1-\exp \left[-\left(\frac{\gamma}{\dot{\varepsilon}^{\beta_{K W W}}}\right)\right]\right\}
$$

Where $\eta_{N}$ is the Newtonian viscosity, $\gamma$ is a fitting parameter related to the critical transition strain rate and $\beta_{K W W}$ is the stretching exponent determining the slope of the non-Newtonian viscosity as a function of strain rate. Generally, the value of $\beta_{K W W}$ is in the range from 0 to 1 . One can obtain the master curve of normalized viscosity $\eta / \eta_{N}$ by horizontal shift of the data obtained at different temperatures. As illustrated by Fig. 2(c), a master curve of normalized viscosity at the reference temperature $T_{r e f}=440 \mathrm{~K}$ was obtained. The horizontal shift leads to a fairly good agreement with the experimental 
135 data. The value of $\beta_{K W W}$ for $\mathrm{La}_{30} \mathrm{Ce}_{30} \mathrm{Ni}_{10} \mathrm{Al}_{20} \mathrm{Co}{ }_{10} \mathrm{HE}-\mathrm{MG}$ is equal to 0.58 . The values

136 of $\beta_{K W W}$ in some HE-MGs and MGs are displayed in Fig. 2(d) (experimental results of 137 other MGs were obtained from literature ${ }^{12,13,31-36}$ ). One can see that the value of $\beta_{K W W}$ 138 in HE-MGs is approximately equal to 0.6 while that of MGs ranges from 0.7 to 0.9 .

139 Therefore, the value of $\beta_{K W W}$ in HE-MGs is significantly lower than the average value 140 found in MGs. It is reasonable to conclude that the degree of deviation from the 141 Newtonian behavior in this HE-MGs is lower in most MGs, which may be due to the 142 high configurational entropy effect on viscous flow.

143 In addition to strain rate jumps, stress relaxation and creep are also powerful methods 144 to explore the anelastic deformation and structural/dynamic heterogeneity of MGs ${ }^{37}$. 145 The mechanical response of HE-MGs under constant stress (creep) or strain (stress 146 relaxation) could be well described by various relaxation events ${ }^{38-40}$. And the evolution 147 of these relaxation events includes various characteristic relaxation times and activation 148 barriers. The width of such distribution of characteristic relaxation times can be 149 assessed by the value of the exponent $\beta_{K W W}{ }^{37}$. In other words, the exponent $\beta_{K W W}$ is an 150 indicator of the structural/dynamic heterogeneity of HE-MGs. A larger $\beta_{K W W}$ 151 corresponds to less heterogeneity. The stretched exponential relaxation has been 152 explained by two limiting scenarios: one is the "homogeneous", all of the particles in 153 the system relax identically but by an intrinsically nonexponential process; the other is 154 the "heterogeneous", the superposition of different simple exponential relaxations with 155 different relaxation times ${ }^{41}$. It is worth mentioning that MGs exhibit various values of $156 \beta_{K W W}$ under the different mechanical simulations, which provides a novel insight into 157 exploring the evolution of structural/dynamic heterogeneity. 
(a)

(c)
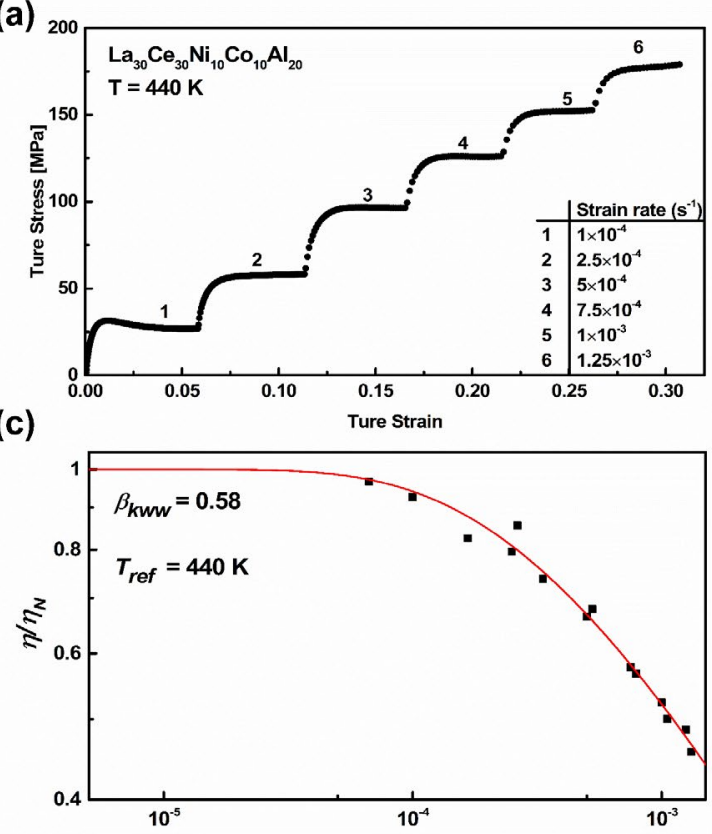

(b)

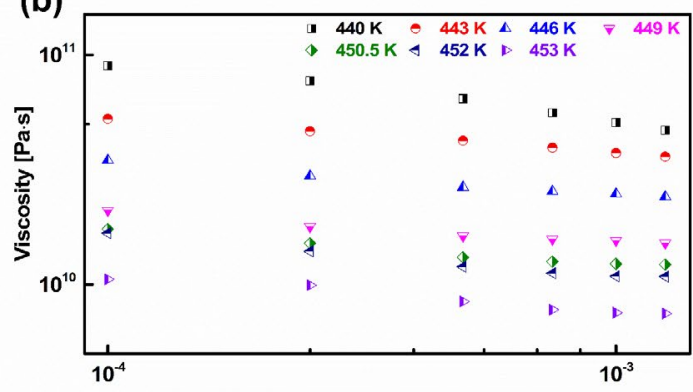

(d)

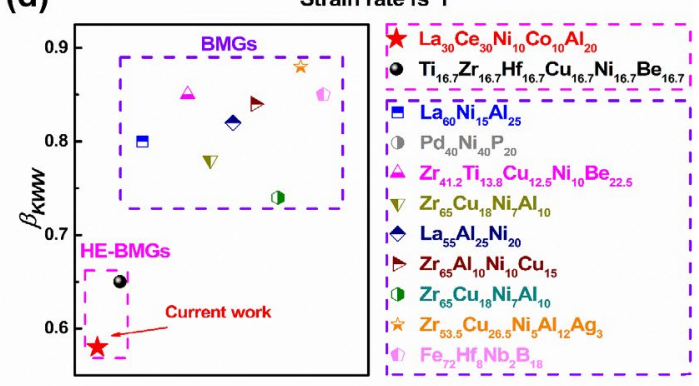

158

159

160

161

162

163

164

165

166

167

168

169

\section{5}

Fig.2 (a) Typical true stress-true strain curve of $\mathrm{La}_{30} \mathrm{Ce}_{30} \mathrm{Ni}_{10} \mathrm{Al}_{20} \mathrm{Co}{ }_{10} \mathrm{HE}-\mathrm{MG}$ during tensile strain rate jump tests at $440 \mathrm{~K}$. The strain rates range from $1 \times 10^{-4}$ to $1.25 \times 10^{-3}$;

(b) Viscosity as a function of strain rate at various temperatures; (c) Master curve of normalized viscosity $\eta / \eta_{N}$ and the fitting curve using Eq. (1), the reference temperature is $440 \mathrm{~K}$; (d) KWW parameter $\beta_{K W W}$ of the normalized viscosity master curve for typical MGs and for HE-MGs.

Transition state theory, which is extensively used to describe the correlation between steady state flow stress and strain rate, was proposed by Turnbull and extended by Spaepen in the framework of the free volume model ${ }^{29}$. Under the situation of uniaxial tension, the transition from non-Newtonian flow to Newtonian flow in typical MGs can be described as ${ }^{29}$ :

$$
\dot{\varepsilon}=2 c_{f} \vartheta_{D} \exp \left(\frac{-\Delta G}{k T}\right) \sinh \left(\frac{\sigma V}{2 \sqrt{3 k T}}\right)=\dot{\varepsilon}_{0} \sinh \left(\frac{\sigma V}{2 \sqrt{3 k T}}\right)
$$

170 where $c_{f}$ is the concentration of activated defects, $\vartheta_{D}$ is the Debye frequency of $10^{13}$

$171 \mathrm{~s}^{-1}, \Delta G$ is the apparent activation energy, $V$ is the apparent characteristic activation 172 volume, and $\dot{\varepsilon}_{0}$ is the temperature-dependent rate factor. Fig. 3(a) displays the steady 173 state flow stress versus strain rate, the solid curves correspond to Eq. (2) with the values 174 of activation volume $V$ fitted from the data at various temperatures. The activation 175 volume was about $0.19 \mathrm{~nm}^{3}$ at $440 \mathrm{~K}$, while it increased to $0.53 \mathrm{~nm}^{3}$ at $453 \mathrm{~K}$, this 176 change reflects a drastic uptrend with temperature. As seen in Fig. 2(b), the viscosity 177 decreases as the test temperature increases, introducing a higher flow capacity and a 
178 larger activation volume at higher temperatures. The above outcomes are consistent 179 with those in various MGs ${ }^{12,13}$. Considering an average atomic volume of $\Omega_{f}=0.013$

$180 \mathrm{~nm}^{3}$, the activation volume during plastic deformation of the $\mathrm{La}_{30} \mathrm{Ce}_{30} \mathrm{Ni}_{10} \mathrm{Al}_{20} \mathrm{Co} 10 \mathrm{HE}-$ 181 MG corresponds to about 20 42 atoms ${ }^{33}$.

182 Previous literature has reported the effects of substituting one element by another 183 with a near-equal atomic radius (such as the substitution of Co by Ni) ${ }^{7,42}$. Such 184 substitution introduces a noteworthy influence on the configurational entropy, which 185 provides a novel insight into exploring the effect of configurational entropy on high 186 temperature deformation of MGs. We selected three MGs with different configurational 187 entropy, i.e., $\mathrm{La}_{60} \mathrm{Ni}_{15} \mathrm{Al}_{25}$, $\mathrm{La}_{30} \mathrm{Ce}_{30} \mathrm{Al}_{15} \mathrm{Co} 25$ and $\mathrm{La}_{30} \mathrm{Ce}_{30} \mathrm{Ni}_{10} \mathrm{Al}_{20} \mathrm{Co} 10 \mathrm{HE}-\mathrm{MG}$, to 188 clarify the correlation between configurational entropy and activation volume. The 189 configurational entropy $S_{c}$ of MGs could be directly estimated as $S_{c}=$ $190-R \sum_{j=1}^{n} x_{j} \ln \left(x_{j}\right)$. One can obtain that the configurational entropy is $0.94 R, 1.35 R$, 191 and $1.50 R$ for $\mathrm{La}_{60} \mathrm{Ni}_{15} \mathrm{Al}_{25}, \mathrm{La}_{30} \mathrm{Ce}_{30} \mathrm{Al}_{15} \mathrm{Co} 25$, and $\mathrm{La}_{30} \mathrm{Ce}_{30} \mathrm{Ni}_{10} \mathrm{Al}_{20} \mathrm{Co} 10$, respectively. 192 Fig. 3(b) shows the evolution of activation volume $V$ as a function of normalized 193 temperature $T / T_{\alpha}$. Interestingly, the activation volume of the La-based HE-MG is 194 significantly smaller than that of the other La-based MGs. It should be pointed out that 195 a smaller activation volume corresponds to less atoms contributing to plastic 196 deformation. It is reasonable to speculate that high configurational entropy promotes 197 small activation volume. In parallel, the Gibbs free energy plays a key role as driving 198 force to activate the atomic rearrangements ${ }^{29}$. The introduction of high configurational 199 entropy reduces the Gibbs free energy, which confirms the correspondence between 200 high configurational entropy and small activation volume ${ }^{43}$. 

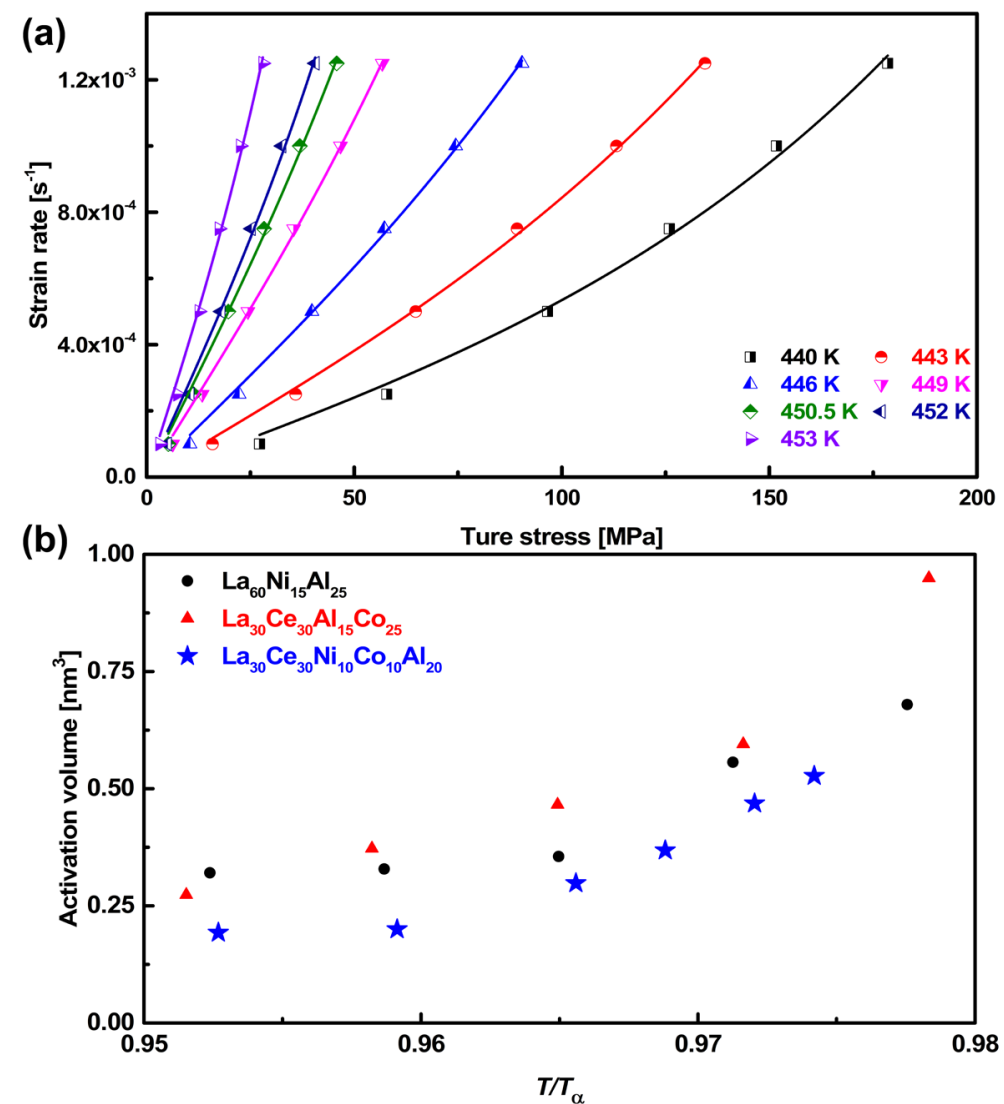

202 Fig.3 (a)Variation of the strain rate with the steady stress in $\mathrm{La}_{30} \mathrm{Ce}_{30} \mathrm{Ni}_{10} \mathrm{Al}_{20} \mathrm{Co}_{10} \mathrm{HE}-$ 203 MG and the fitting curves using Eq. (2); (b) Normalized temperature dependence of 204 activation volume in $\mathrm{La}_{60} \mathrm{Ni}_{15} \mathrm{Al}_{25} \mathrm{MG}, \mathrm{La}_{30} \mathrm{Ce}_{30} \mathrm{Al}_{15} \mathrm{Co} 25 \quad \mathrm{MG}$ and $205 \mathrm{La}_{30} \mathrm{Ce}_{30} \mathrm{Ni}_{10} \mathrm{Al}_{20} \mathrm{Co} 10 \mathrm{HE}-\mathrm{MG}$.

206 From the perspective of potential energy landscape, the slow $\beta$ relaxation is 207 associated with atomic transition processes between subbasins ${ }^{44}$. In addition, the slow $208 \beta$ relaxation is the trigger to activate the plastic strain during the plastic deformation ${ }^{45}$. 209 Assuming that the applied stress slopes the potential energy landscape, resulting in a 210 lower potential barrier for the transition to a stable configuration, with the increase of 211 the stress more atoms trapped in the basins can be activated by thermal and mechanical 212 stimulation thus triggering the plastic deformation.

213 It is well known that La- and Ce-based MGs display a distinct peak of slow $\beta$ 214 relaxation while CuZr-based MGs exhibit a weaker slow $\beta$ relaxation, the latter 215 observed as an "excess wing" 1 . The activation volume of high temperature deformation 216 in CuZr-based MGs has been reported to range from $0.15 \mathrm{~nm}^{3}$ to $0.35 \mathrm{~nm}^{3} 46$. 217 Significantly, the activation volumes and the concentration of flow defects of La-based 218 MGs in the current work are higher than the ones found in CuZr-based MGs. In the 
219 literature, MGs have been modelled as constituted by soft "liquid-like" and hard "solid220 like" regions ${ }^{47}$. The soft "liquid-like" regions, where the deformation units are easier 221 to activate, possess low density, low elastic modulus and high atomic mobility 222 associated with high flow capacity. It is well accepted that the slow $\beta$ relaxation, 223 originated from the string-like atoms movement in "soft" regions, is closely associated 224 with the structural heterogeneity $1,2,27,48$. Therefore, more atoms may be involved in 225 response to thermal or mechanical stimulations and contribute to the plastic 226 deformation in MGs with obvious slow $\beta$ relaxation. It is then reasonable that MGs 227 possessing apparent slow $\beta$ relaxation show higher activation volumes, which is in good 228 agreement with the experimental results. Fig. 4 is a schematic diagram of activation 229 volumes in MGs and HE-MGs showing a pronounced slow $\beta$ relaxation. From the 230 results shown in this work, less atoms are activated in $\mathrm{La}_{30} \mathrm{Ce}_{30} \mathrm{Ni}_{10} \mathrm{Al}_{20} \mathrm{Co} 10 \mathrm{HE}-\mathrm{MG}$. 231 Nevertheless, knowledge of the distribution of deformation units (liquid-like regions) 232 is absent, which makes difficult to establish the correlation between structural 233 heterogeneity and high configurational entropy.
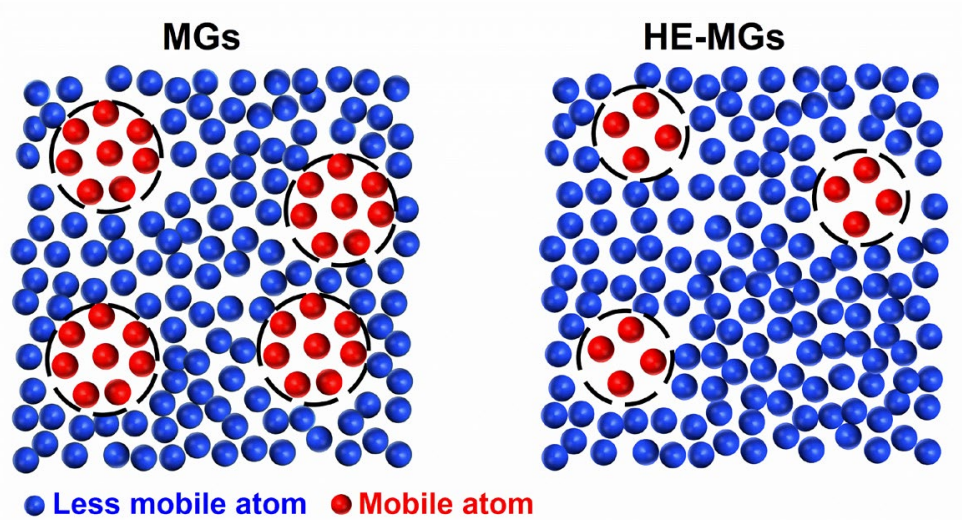

Fig.4 Schematic diagram of activation volumes in MGs and HE-MGs with a pronounced $\beta$ relaxation.

In summary, the high temperature deformation behavior and the effect of high configurational entropy on the high temperature deformation mechanism of $\mathrm{La}_{30} \mathrm{Ce}_{30} \mathrm{Ni}_{10} \mathrm{Al}_{20} \mathrm{Co} 10 \mathrm{HE}-\mathrm{MG}$ were discussed in the framework of the free volume model. The main results are summarized as follows:

- $\mathrm{La}_{30} \mathrm{Ce}_{30} \mathrm{Ni}_{10} \mathrm{Al}_{20} \mathrm{Co}{ }_{10} \mathrm{HE}-\mathrm{MG}$ with a pronounced slow $\beta$ relaxation is a suitable candidate to elucidate the effects of both high configurational entropy and slow $\beta$ relaxation on the mechanical behavior of MGs.

\section{- Different from conventional La-based MGs, a weaker deviation from Newtonian}


behavior is found in the HE-MG, which can be ascribed to higher configurational entropy.

- Compared with conventional La-based MGs, in La30 $\mathrm{Ce}_{30} \mathrm{Ni}_{10} \mathrm{Al}_{20} \mathrm{Co}{ }_{10} \mathrm{HE}-\mathrm{MG}$

248 the high configurational entropy is related to a small activation volume of thehigh

249 temperature deformation behavior.

250 Acknowledgements

251 This work is supported by the National Natural Science Foundation of China (NSFC) 252 (Grant No. 51971178) and Natural Science Basic Research Plan for Distinguished 253 Young Scholars in Shaanxi Province (Grant No. 2021JC-12). E. Pineda and D. Crespo 254 acknowledge financial support from MICINN (grant FIS2017-82625-P) and 255 Generalitat de Catalunya (grant 2017SGR0042). The investigation of Y. J. Duan is 256 sponsored by Innovation Foundation for Doctor Dissertation of Northwestern 257 Polytechnical University (No. CX202031). The investigation of L.T. Zhang is 258 sponsored by Innovation Foundation for Doctor Dissertation of Northwestern 259 Polytechnical University (No. CX2021015).

\section{Data availability statement}

261 The data that supports the findings of this study are available within the article.

\section{References}

2631 J.C. Qiao, Q. Wang, J.M. Pelletier, H. Kato, R. Casalini, D. Crespo, E. Pineda, 264 Y. Yao, and Y. Yang, Prog. Mater. Sci. 104, 250 (2019).

2652 W.H. Wang, Prog. Mater. Sci. 106, 100561 (2019).

2663 A. L. Greer, Y. Q. Cheng, and E. Ma, Mater. Sci. Eng., R 74 (4), 71 (2013).

2674 Y. F. Ye, Q. Wang, J. Lu, C. T. Liu, and Y. Yang, Mater, Today 19 (6), 349 268 (2016);

2695 P. Sathiyamoorthi and H.S. Kim, Prog. Mater. Sci., 100709 (2020).

2706 A. Takeuchi, N. Chen, T. Wada, Y. Yokoyama, H. Kato, A. Inoue, and J. W. Yeh, $271 \quad$ Intermetallics 19 (10), $1546(2011)$;

2727 T. Wada, J. Jiang, K. Yubuta, H. Kato and A. Takeuchi, Materialia 7, 100372 273 (2019). 
2748 S. F. Zhao, G. N. Yang, H. Y. Ding, and K. F. Yao, Intermetallics 61, 47 (2015).

2759 C. E. Packard and C. A. Schuh, Acta Materialia 55 (16), 5348 (2007).

$27610 \quad$ S. Takeuchi and K. Edagawa, Prog. Mater. Sci. 56 (6), 785 (2011).

$27711 \quad$ M. Ghidelli, H. Idrissi, S. Gravier, J.J. Blandin, J.P. Raskin, D. Schryvers, and 278 T. Pardoen, Acta Mater 131, 246 (2017).

27912 J. Lu, G. Ravichandran, and W. L. Johnson, Acta Mater 51 (12), 3429 (2003).

28013 Y. Kawamura, T. Nakamura, H. Kato, H. Mano, and A. Inoue, Mater. Sci. Eng., $281 \quad$ A 304 (none), 674 (2001).

28214 P. H. Cao, M. P. Short, and S. Yip, Proc. Natl. Acad. Sci. 114 (52), 13631 283 (2017);

28415 J. C. Qiao, Y. J. Wang, L. Z. Zhao, L. H. Dai, D. Crespo, J.M. Pelletier, L.M. 285 Keer, and Y. Yao, Phys. Rev. B 94 (10), 104203 (2016);

28616 A. Makarov, Y. P. Mitrofanov, G. Afonin, N. Kobelev and V. Khonik, Scr. 287 Mater. 168, 10 (2019).

28817 B. Y. Cui, Z. Evenson, B. Fan, M. Z. Li, W. H. Wang, and A. Zaccone, Phys. 289 Rev. B 98 (14), 144201 (2018);

29018 F. Zhu, H. K. Nguyen, S. X. Song, Daisman P. B. Aji, A. Hirata, H. Wang, K. 291 Nakajima, and M. W. Chen, Nat. Commun. 7 (1), 11516 (2016).

29219 H. B. Yu, W. H. Wang, H. Y. Bai, Y. Wu, and M.W. Chen, Phys. Rev. B 81 $293 \quad$ (22), $220201(2010)$.

29420 Z. Yong, T.T. Zuo, T. Zhi, M.C. Gao, K.A. Dahmen, P.K. Liaw, and Z. P. Lu, 295 Prog. Mater. Sci. 61, 1 (2014).

29621 H. Yin, Y. Huang, D. Daisenberg, P. Xue, S. Jiang, W. Ru, S. Jiang, Y. Bao, X. 297 Bian, X. Tong, H. Shen, and J. F. Sun, Scr. Mater. 163, 29 (2019);

298 W. Jiang and B. Zhang, J. Appl. Phys. 127 (11), 115104 (2020);

29923 L. T. Zhang, Y. J. Duan, T. Wada, H. Kato, J. M. Pelletier, D. Crespo, E. Pineda, 300 and J. C. Qiao, J. Mater. Sci. Technol. 83, 248 (2021).

$30124 \quad$ J. Kim, H.S. Oh, J. Kim, C.W. Ryu, G.W. Lee, H.J. Chang, and E.S. Park, Acta 
Mater 155, 350 (2018).

Q. Zhou, Y. Du, W. Han, Y. Ren, H. Zhai, and H. F. Wang, Scr. Mater. 164, $121(2019)$. (2013). 095508 (2012). 1257 (2006).

F. Spaepen, Acta Metall. 25 (4), 407 (1977). 306, 735 (2001). Mater 98, 43 (2015). (2020);

C. Zhang, J. C. Qiao, J. M. Pelletier, and Y. Yao, Scr. Mater. 113, 180 (2016); (2015). M. Atzmon, Acta Mater 164, 165 (2019). Jiang Wang, Intermetallics 125, 106922 (2020).

$328 \quad 40$ Zhen Lu, Wei Hua Wang, and Hai Yang Bai, Sci. China Mater. 58 (2), 98 329 (2015). 

(3), 590 (1998). J. S. Lee, H. S. Oh, W. Kim, C.W. Ryu, J. Y. Kim, H. J. Chang, J. L. Gu, K. F. Yao, B. S. Murty, and E. S. Park, Materialia 9, 100505 (2020).

33443 R. Busch, J. Schroers, and W. H. Wang, MRS Bulletin 32 (8), 620 (2007).

33544 P. G. Debenedetti and F. H. Stillinger, Nature 410 (6825), 259 (2001).

33645 Z. Lu, W. Jiao, W. H. Wang, and H. Y. Bai, Phys. Rev. Lett. 113 (4), 045501 337 (2014).

$33846 \quad$ Y. J. Huang, J. Shen, Y. Sun, J. F. Sun, and John J. J. Chen, J. Alloys Compd. $339 \quad \mathbf{5 0 4}, \mathrm{S} 82(2010)$.

34047 W. H. Wang, J. Appl. Phys. 110 (V110N5), 053521 (2011).

34148 H. B. Yu, X. Shen, Z. Wang, L. Gu, W. H. Wang, and H.Y. Bai, Phys. Rev. 342 Lett. 108 (1), 015504 (2012). 